\title{
A COHOMOLOGY FOR FOLIATED MANIFOLDS
}

\author{
BY JAMES L. HEITSCH ${ }^{1}$
}

Communicated by S. S. Chern, May 1, 1973

1. Introduction. Let $M$ be a connected manifold and $\tau$ a foliation on $M$. $\tau$ is then an involutive subbundle of $T M$, the tangent bundle of $M$. Denote by $v$ the normal bundle to $\tau, v=T M / \tau$. We denote sections of a bundle $P$ over $M$ by $\Gamma(P)$. All manifolds, bundles and maps are assumed to be $C^{\infty}$.

There is a canonical connection $\nabla$ on $v$ which is flat along $\tau[\mathbf{B}]$. Consider the complex

$$
\Gamma(v) \stackrel{d}{\longrightarrow} \Gamma\left(v \otimes \Lambda^{1} \tau^{*}\right) \stackrel{\hat{d}}{\longrightarrow} \Gamma\left(v \otimes \Lambda^{2} \tau^{*}\right) \stackrel{d}{\longrightarrow} \cdots,
$$

where $\tau^{*}$ is the cotangent bundle to the foliation and

$$
\begin{aligned}
\hat{d}(\sigma) & \left(X_{1}, \ldots, X_{k+1}\right) \\
= & \sum_{1 \leqq i \leqq k+1}(-1)^{i} \nabla_{X_{i}}\left(\sigma\left(X_{1}, \ldots, \hat{X}_{i}, \ldots, X_{k+1}\right)\right) \\
& +\sum_{1 \leqq i<j \leqq k+1}(-1)^{i+j+1} \sigma\left(\left[X_{i}, X_{j}\right], X_{1}, \ldots, \hat{X}_{i}, \ldots, \hat{X}_{j}, \ldots, X_{k+1}\right)
\end{aligned}
$$

for $\sigma \in \Gamma\left(v \otimes \Lambda^{k} \tau^{*}\right), X_{1}, \ldots, X_{k+1} \in \Gamma(\tau)$.

Since the curvature tensor of $\nabla$ restricted to $\tau$ is identically zero we have that $\hat{d} \circ \hat{d}=0$. Denote the homology of this complex by $F^{*}(\tau ; v)$. This is the cohomology of the Lie algebra of vector fields tangent to the foliation with coefficients in sections of the normal bundle, the representation being given by the connection [GF].

In general the groups $F^{k}(\tau ; v)$ are not finitely generated (the complex is not elliptic) but they satisfy the following.

(i) $F^{*}$ is a functor from the category of foliated manifolds and transverse maps to the category of abelian groups and homomorphisms.

(ii) If $f: N \rightarrow M$ is an embedded transverse submanifold, we can define relative cohomology groups $F^{*}(\tau ; v, f)$ and obtain the usual long exact sequence.

(iii) $F^{*}$ is an invariant of the diffeomorphism type of the foliation. However, $F^{*}$ is not an invariant of the integrable homotopy type of the foliation when $M$ is an open manifold.

2. Interpretation of $F^{1}(\tau ; v)$. Fix a Riemannian metric on $M$ and think

AMS (MOS) subject classifications (1970). Primary 14F05, 55B30, 57D30.

${ }^{1}$ Supported in part by National Science Foundation grant GP-34785X. 
of $v$ as all tangent vectors normal to $\tau$. We then have projection operators $\pi: T M \rightarrow \tau$ and $\pi^{\perp}: T M \rightarrow v$. We can view $\Gamma\left(v \otimes \tau^{*}\right)$ as infinitesimal deformations of $\tau$ as follows: Let $\tau_{s}, s \in \boldsymbol{R}$, be a differentiable family of codimension $q$ subbundles of $T M$ with $\tau_{0}=\tau$ and let $\pi_{s}$ and $\pi_{s}^{\perp}$ be the associated projection operators. For each $X \in \tau_{0}$ define

$$
\sigma(X)=d /\left.d s\left(\pi_{s}(X)\right)\right|_{s=0} .
$$

We call $\sigma$ the infinitesimal deformation associated to the family $\tau_{s}$ and note that $\sigma \in \Gamma\left(v \otimes \tau^{*}\right)$.

For each $s \in R$, let $\Phi_{s}(X, Y)=\pi_{s}^{\perp}\left(\left[\pi_{s} X, \pi_{s} Y\right]\right)$.

LEMMA. (i) $\Phi_{s}$ is an exterior 2 form on TM.

(ii) $\tau_{s}$ is involutive if and only if $\Phi_{s} \equiv 0$.

(iii) $\hat{d} \sigma(X, Y)=d /\left.d s\left(\Phi_{s}(X, Y)\right)\right|_{s=0}$.

Proposition 1. Let $\tau_{s}$ be a differentiable family of subbundles of TM all of which are foliations. Let $\sigma$ be the associated infinitesimal deformation. Then $\hat{d} \sigma=0$.

Proposition 2. Let $\phi_{s}, s \in \boldsymbol{R}$, be a flow on $M, X$ the associated vector field and $\tau_{0}$ a foliation. For each $s \in \boldsymbol{R},\left(\phi_{s}\right)_{*} \tau_{0}$ is a foliation on $M$ denoted $\tau_{s}$. Let $\sigma$ be the associated infinitesimal deformation. Then $\sigma=\hat{d}\left(\pi_{0}^{\perp} X\right)$.

Note that any element $X \in \Gamma(v)$ generates a local flow whose associated infinitesimal deformation is $\hat{d} X$. Thus we may view $F^{1}(\tau ; v)$ as infinitesimal deformations of the foliation $\tau$, modulo trivial deformations.

Question. Given $\alpha \in F^{1}(\tau ; v)$, under what conditions does there exist an element $\sigma \in \alpha$ which comes from a deformation of $\tau$ through foliations?

EXAMPLE. Constant slope foliations on $T^{2}$. Let $T^{2}=\boldsymbol{R}^{2} / \boldsymbol{Z}^{2}$ and denote by $\tau_{a}$ the foliation of $T^{2}$ given by all straight lines of slope $a$.

THEOREM 1. If a is rational then $F^{0}\left(\tau_{a} ; v_{a}\right) \cong C^{\infty}\left(S^{1}\right), F^{1}\left(\tau_{a} ; v_{a}\right) \cong C^{\infty}\left(S^{1}\right)$ and each element of $F^{1}\left(\tau_{a} ; v_{a}\right)$ can be realized as the associated infinitesimal deformation of a differentiable family of foliations.

Recall that an irrational number $a$ is not a Liouville number provided there is a positive integer $p$ and $\varepsilon>0$ such that $|a-n / m|>\varepsilon(|m|+|n|)^{-p}$ for $m$ and $n$ sufficiently large integers.

THEOREM 2. If a is irrational then $F^{0}\left(\tau_{a} ; v_{a}\right) \cong \boldsymbol{R}$. If a is not a Liouville number then $F^{1}\left(\tau_{a} ; v_{a}\right) \cong \boldsymbol{R}$ and each element of $F^{1}\left(\tau_{a} ; v_{a}\right)$ can be realized as the associated infinitesimal deformation of a differentiable family of foliations.

See $[\mathbf{H}]$.

3. The complex restricted to a leaf. Let $L$ be a leaf of a foliation $\tau$ on $M$ 
and denote by $v$ the normal bundle of $\tau$ restricted to $L$. Consider the complex

$$
\Gamma(v) \stackrel{\hat{d}}{\longrightarrow} \Gamma\left(v \otimes \Lambda^{1} T^{*} L\right) \stackrel{\hat{d}}{\longrightarrow} \Gamma\left(v \otimes \Lambda^{2} T^{*} L\right) \stackrel{\hat{d}}{\longrightarrow} \cdots,
$$

where $\hat{d}$ is defined as above. Again $\hat{d}^{2}=0$ and we denote the resulting groups by $F^{*}(L)$.

THEOREM 3. $F^{*}(L)$ is isomorphic to $H^{*}\left(L ; \boldsymbol{R}_{\sim}^{q}\right)$, the cohomology of $L$ with coefficients in $\boldsymbol{R}_{\sim}^{q}(q=\operatorname{dim} v)$ twisted over the linear holonomy of the foliation $\tau$.

We prove this by noting that the linear holonomy of the foliation is the holonomy of the canonical connection on $v$. We then show that the complex $\left\{\Gamma\left(v \otimes \Lambda^{k} T^{*} L\right), \hat{d}\right\}$ is isomorphic to the complex $\left\{\mathscr{A}_{\pi_{1}(L)}\left(\tilde{L} ; \boldsymbol{R}_{\sim}^{z}\right), d\right\}$, the de Rham complex of $\boldsymbol{R}_{\sim}^{q}$ valued forms on the simply connected covering space $\tilde{L}$ of $L$ which satisfy:

$$
\left(\sigma^{*} \omega\right)\left(Y_{1}, \ldots, Y_{k}\right)=h\left(\sigma^{-1}\right)\left(\omega\left(Y_{1}, \ldots, Y_{k}\right)\right)
$$

where $\omega$ is an $R_{\sim}^{q}$ valued $k$-form on $\tilde{L}, Y_{1}, \ldots, Y_{k} \in \Gamma(T \tilde{L}), \sigma \in \pi_{1}(L)$ and acts on $\tilde{L}$, by deck transformations, and $h: \pi_{1}(L) \rightarrow \operatorname{GL}(q, R)$ is the holonomy representation.

\section{REFERENCES}

[B] R. Bott, On a topological obstruction to integrability, Proc. Sympos. Pure Math., vol. 16, Amer. Math. Soc., Providence, R.I., 1970, pp. 127-131. MR 42 \#1155.

[GF] I. M. Gel'fand and D. B. Fuchs, Cohomologies of the Lie algebra of tangent vector fields on a smooth manifold, Funkcional. Anal. i Priložen. 3 (1969), no. 3, 32-52. (Russian) MR 41 \# 1067.

[H] C. S. Herz, Functions which are divergences, Amer. J. Math. 92 (1970), 641-656. MR 44 \# 7590 .

Department of Mathematics, University of California, Berkeley, California 94720

Current address: Department of Mathematics, University of California, Los Angeles, California 90024 